\section{ESTUDIO BROMATOLÓGICO DEL FRUTO Y PALMA DE LA ESPECIE ARBÓREA EUTERPE PRECATORIA, PALMERA DE ASAÍ, IXIAMAS, DEPARTAMENTO DE LA PAZ, BOLIVIA}

Full original article
Received 04052021

Accepted 12082021

Published 12302021

Vol. 38, No.5, pp. 223-232, Nov./Dic.2021

Revista Boliviana de Química

$38(5), 223-232$, Nov./Dec. 2021

Bolivian Journal of Chemistry

DOI : $10.34098 / 2078-3949.38 .5 .4$

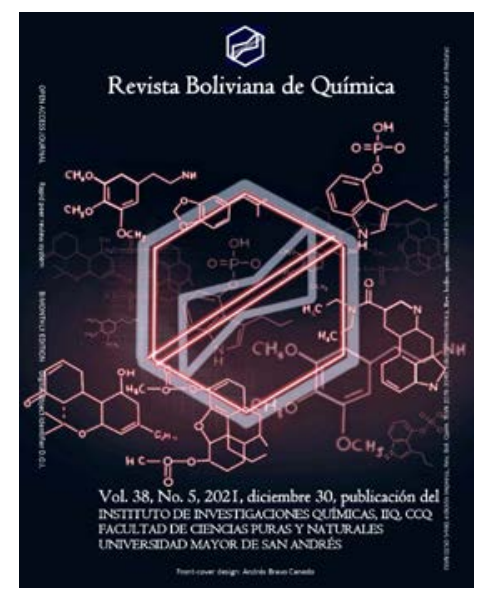

Galia Chávez Cury' ${ }^{1, *}$, Willy J. Rendón Porcel ${ }^{1}$, J. Mauricio Peñarrieta ${ }^{1}$, María del Carmen Abela Gisbert ${ }^{2}$

${ }^{1}$ Food Chemistry Laboratory, Instituto de Investigaciones Químicas IIQ, Chemical Sciences Department, Facultad de Ciencias Puras y Naturales FCPN, Universidad Mayor de San Andrés UMSA, P.O. Box 303, Calle Andrés Bello s/n, Ciudad Universitaria Cota Cota, phone +59122795878, La Paz, Bolivia, jmpenarrieta1@umsa.bo, pamollinedo@umsa.bo, www.iiq.umsa.bo

2Laboratorio de Análisis Sensorial, Carrera de Nutrición y Dietética, Facultad de Medicina, Universidad Mayor de San Andrés UMSA, Av. Saavedra 2246, Miraflores, phone +59122612371, La Paz, Bolivia, carrera.medicina@umsalud.edu.bo,www.fment.umsa.bo

Keywords: Palm, Asaí, Euterpe precatoria, palm heart, Bromatological, Antioxidants, Bolivia

Palabras clave: Palmeras, Asaí, Euterpe precatoria, Palmito, Bromatológico, Antioxidantes, Bolivia

\title{
ABSTRACT
}

The study of the nutritional content of the edible parts (fruit and hearts of palm) of the tree species Euterpe precatoria (Arecaceae) or Asaí Palm was carried out. The analyses demonstrated the high nutritional value of the species. The samples were collected in the town of Ixiamas and its surroundings (North of the Department of La Paz). The energy content of the fruit is approximately $515.4 \mathrm{kcal}$ and of the palm heart ranges between 349.7 $361.8 \mathrm{kcal}$. The protein content of the fruit and of the palm heart ranges between $8.4-8.8 \%$ and $21.4-35.5 \%$ respectively. The Asaí fruit has a high content of Vitamin A, of: 442.47 ug / 100g. Palmito and fruit have high content of calcium, magnesium and potassium. It is important to note that an important antioxidant capacity was also determined in the Asaí samples.

*Correspondent autor: galiachavez1972@gmail.com 


\section{RESUMEN}

Se realizó el estudio del contenido nutricional de las partes comestibles (fruto y palmito) de la especie Euterpe precatoria (Arecaceae) o Palmera Asaí. Los análisis demostraron el alto valor nutritivo de la especie. Las muestras se recolectaron en la localidad de Ixiamas y sus alrededores (Norte del Depto. de La Paz). El contenido energético del fruto es de aproximadamente $515.4 \mathrm{kcal}$ y del palmito oscila entre 349.7 - $361.8 \mathrm{kcal}$. El contenido de proteínas del fruto y del palmito oscila entre 8.4 - 8.8 \% y 21.4- $35.5 \%$ respectivamente. El fruto del Asaí tiene un alto contenido en Vitamina A, de: 442.47 ug/100g. Palmito y fruto presentan contenidos altos en calcio, magnesio y potasio. Es importante indicar que también se determinó una capacidad antioxidante importante en las muestras de Asaí.

\section{INTRODUCCIÓN}

Bolivia es un país ecológicamente mega diverso, dando lugar a una gran variedad de ecosistemas [1]. Entre éstos se encuentra la región Amazónica, compartida con siete países sudamericanos. La Amazonia de Bolivia abarca los Departamentos del Beni, Pando y parte de los departamentos de La Paz, Santa Cruz y Cochabamba, donde viven cientos de etnias poseedoras de un conocimiento milenario [2].

La Provincia Abel Iturralde, al norte del departamento de La Paz, tiene como capital a la localidad de Ixiamas, puerta norte a la Amazonía Boliviana. Es una zona tropical rica en especies alimenticias, conocidas a nivel local, y en menor grado a nivel regional y nacional, nuestro objetivo es valorizar dichas especies en cuanto a su contenido energético, y su valor en macro y micronutrientes.

La gran variedad alimentaria de la zona permite a los lugareños disponer a discreción de los recursos alimenticios para satisfacer sus necesidades nutritivas, quedando esta disponibilidad sujeta, al uso sostenible que se les dé a estos recursos.

Se conoce el alto valor nutritivo de las palmeras desde épocas remotas en lugares tan diferentes cono el medio y lejano oriente, así como las selvas americanas donde eran bien reputadas como alimentos, siendo estos árboles, además, de las gramíneas y leguminosas, las que proveen productos no solo alimenticios, sino también medicinales. Las poblaciones locales del país aprovechan los productos de las diferentes especies de palmeras: comercializan los frutos, las hojas para el techado de las casas y el palmito en la alimentación.

El palmito de la especie Euterpe precatoria, tiene un gran valor económico por su elevada demanda comercial, aportando un ítem más a los productos para la exportación, con tasas de ingresos económicos importantes a nivel regional y nacional. Pero es importante dar a conocer, que cada vez que se "cosecha el palmito," se elimina totalmente la planta [4], pues presenta un solo brote.

Ante esta acción que lleva a la eliminación de la planta, que da frutos ricos en nutrientes, muy apetecidos por la población boliviana, habría que tomar en cuenta otras especies de palmeras que no presenten un solo tallo como el caso de la palmera Asaí, para que pueda abastecer de palmito a la población, sin mayores problemas para la conservación de la planta [4].

El riesgo de anemia y de hipovitaminosis A en la población infantil de nuestro país repercute en la prolongación de las enfermedades contagiosas, como diarreas e infecciones respiratorias agudas, principales causas de la morbimortalidad en niños [5]. Otro grupo que presenta anemia por deficiencia de hierro, son las mujeres en edad fértil de 15 a 45 años [6].

Bolivia no cuenta con información sobre el contenido nutricional y de metabolitos secundarios de la mayor parte de especies vegetales comestibles. Sin embargo, el Instituto Nacional de Laboratorios de Salud INLASA ha publicado una tabla del contenido nutricional de varias especies vegetales alimenticias. Por otra parte, se cuenta con información bibliográfica de investigaciones de países vecinos como por ejemplo sobre los contenidos nutricionales de especies vegetales alimenticias de la amazonia peruana [7]. Los contenidos nutricionales y de metabolitos secundarios de las especies vegetales alimenticias está en función de los aspectos genéticos, las prácticas culturales, la radiación solar, la disponibilidad de agua, la época del año, la fertilización, la topografía, etc. causando que la composición de una misma variedad de planta cambie de contenidos, según la región geográfica donde se cultive, por lo que no se puede usar una tabla de composición nutricional genérica en todos los países incluso en un mismo país [8].

En este artículo se reporta los resultados del estudio bromatológico y nutricional de los frutos y el palmito de la palmera Euterpe precatoria (Arecaceae) C. Martius (Asaí), con muestras recolectadas en la localidad de Ixiamas (capital de la provincia Abel Iturralde del dpto. de La Paz - Norte Paceño) y sus alrededores. También se realizó una encuesta sobre la degustación del refresco de Asaí, producto elaborado artesanalmente a partir de los frutos.

Downloadable from: Revista Boliviana de Química. http://www.bolivianchemistryjournal.org
224

Volumen 38 N5. Año 2021

http://www.scribd.com/bolivianjournalofchemistry 
Es importante indicar que todas las zonas tropicales de Bolivia, incluido el Norte paceño, presentan altos índices de desnutrición y anemia en la población infantil y de mujeres en de edad fértil.

El objetivo fue obtener información técnica que sirva de base, para el uso adecuado de estas especies botánicas, en la dieta diaria de los habitantes de la zona y otras poblaciones del país. Esto derivaría en un uso racional de los productos alimenticios en particular de la especie, con una consecuente mejora en el desarrollo sostenible de la zona.

Además, se realizó la actividad antioxidante del Asaí fue determinada. Cabe señalar que la especie ha sido declarada en vías de extinción [8].

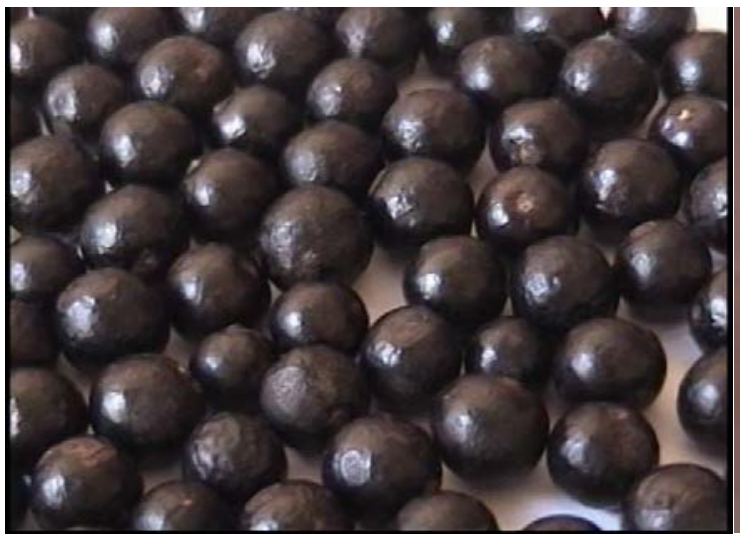

FRUTO: Asaí

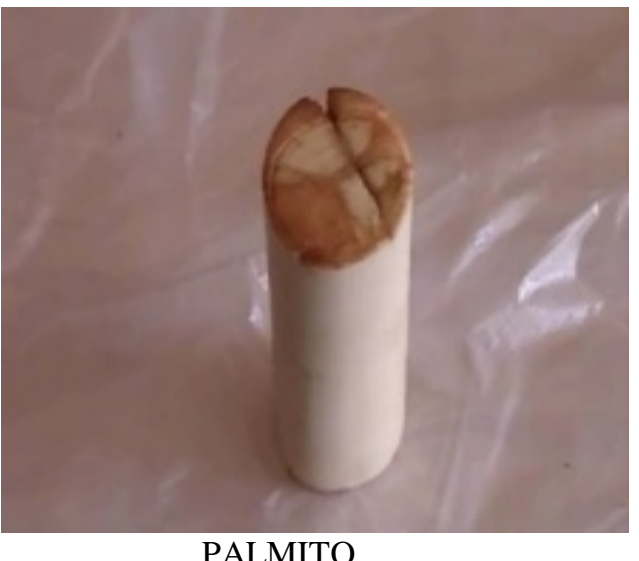

PALMITO

\section{Breve descripción y habitat de la palmera (Euterpe precatoria, Asaí). Características físicas y usos de los frutos y del palmito.}

El Asaí crece en las orillas de los ríos y en lugares temporalmente inundados, "curichis,” (charco grande), medio en de grandes árboles, propios de bosques altos [9]. Es una de las más elegantes y esbeltas palmeras de la selva boliviana. En los últimos años el número de estas palmeras ha ido disminuyendo dramáticamente, debido a la sobre explotación que realizan las empresas envasadoras de palmito [9], pues cada vez que se "cosecha" el palmito, se elimina totalmente la planta [4].

La palmera de Asaí, es una planta inerme (sin espinas), de 15 a $20 \mathrm{~m}$ de alto, con frutos globosos de 1,3 cm de diámetro, epicarpio negro en maduro, mesocarpio jugoso, fibroso de color morado; endocarpio duro, delgado con fibras longitudinales; semilla globosa de $1 \mathrm{~cm}$ de diámetro [9]. Las plantas adultas pueden tener una altura de 30 a $40 \mathrm{~m}$ sobre el suelo con partes expuestas al aire libre [7]. Los frutos de Asaí, se usan para obtener bebidas refrescantes, también el jugo es utilizado para cocinar pescado [9].

El palmito, es el brote o los brotes terminales de diferentes tipos de palmeras. Se extrae del cogollo tierno ubicado al cabo del estípite de la palmera, formado por hojas aún inmaduras, del cual se elimina la corteza y las capas fibrosas y duras de su interior. El palmito es de color blanco marfil, textura suave y flexible, rico en fibras. Muchas palmeras sólo tienen un solo brote y al sacarlo éste, la palmera se muere como es el caso de las palmeras Asaí, Motacú, Majo, etc. En el caso de la palmera Asaí, en general se derriba la misma porque es demasiado alta, también es cortada para si no la cortaban, al sacarle el brote. Esta es la razón por la que esta especie de palmera está en vías de extinción, debido al auge de exportación.

Actualmente hay empresas que exportan palmito, tienen años de trabajo, han realizado siembras especiales de palmeras, como es el caso de la especie Bactris gasipaes H. B. K. (Tembe), que es una palmera con muchos brotes, de tal manera que cosechan palmitos sin mayores problemas, pues son cultivos sostenibles y ecológicos.

\section{EXPERIMENTAL}

\section{Lugar de muestreo}

Las muestras fueron colectadas en la localidad de Ixiamas es una zona de topografía plana, con llanuras extensas susceptibles a inundaciones. Presenta una temperatura promedio anual de $23^{\circ} \mathrm{C}$, una precipitación de $2000 \mathrm{~mm}$, clima tropical húmedo y bosques extensos [1]. Es una zona agrícola, ganadera y maderera. La localidad de Ixiamas

Downloadable from: Revista Boliviana de Química. http://www.bolivianchemistryjournal.org
225

Volumen 38 N5. Año 2021

http://www.scribd.com/bolivianjournalofchemistry 
Galia Chávez Cury et al. RBQ Vol.38, No.5, pp. 223-232, 2021

$\left(13^{\circ} 45^{\prime}\right.$ Sur $68^{\circ} 09^{\prime}$ Oeste) [2] es Capital de la Provincia Abel Iturralde del Dpto. de La Paz, tiene una altitud de

250 msnm y una población aproximad de 2500 habitantes (Com. per.).

\section{Material Vegetal}

Las muestras de palmito y frutos, fueron recolectadas por el Sr. J. Mamani.

\section{A.- Muestras de Palmito de diferentes puntos:}

Muestra 1 (Pal 1) Punto C:

Se tomó la muestra en las tierras (Chaco) del Sr. Jorge Mamani.

Muestra 2 (Pal 2) Punto D:

La muestra fue tomada en las tierras del Sr. Saúl Garret,

Muestra 3 (Pal 3): Punto I

La muestra fue tomada en las tierras del Sr. Saúl Garret

\section{B.- Muestras de los frutos del Asaí: Las muestras se tomaron por la carretera de Ixiamas-Pando} en bosques del Río Undumo (Punto I).

Se recolectaron muestras de frutos de diferentes plantas de Asaí, (Punto I) y se dividieron en 3 partes: Muestra 1 (Asa1), Muestra 2 (Asa 2) y Muestra 3 (Asa 3). Para los análisis se utilizó la parte comestible del fruto (pulpa y cáscara).

La ubicación de los diferentes puntos donde se tomaron y recolectaron las muestras del fruto y palmito se encuentran en la Tabla 1, también se pueden observar los diferentes puntos en imagen satelital (Fig.1). Las imágenes fueron elaboradas por GPS-Bolivia

Tabla 1. Ubicación de los diferentes puntos de recolecta de palmito y frutos.

\begin{tabular}{lcccc}
\hline Nombre & Latitud & Longitud & Altitud(m) & Observaciones \\
\hline Punto C & $-1347.39300^{\prime}$ & -68 10.64800' & 286 & Pal.1, Pal. 2 y Pal.3 \\
Punto D & $-1346.63900^{\prime}$ & $-6809.17800^{\prime}$ & 263 & Próximo al Río Undumo \\
Punto I & $-1345.12978^{\prime}$ & $-6820.72525^{\prime}$ & $\mathrm{n} / \mathrm{d}$ & Asa 1, Asa 2 y Asa 3 \\
\hline
\end{tabular}

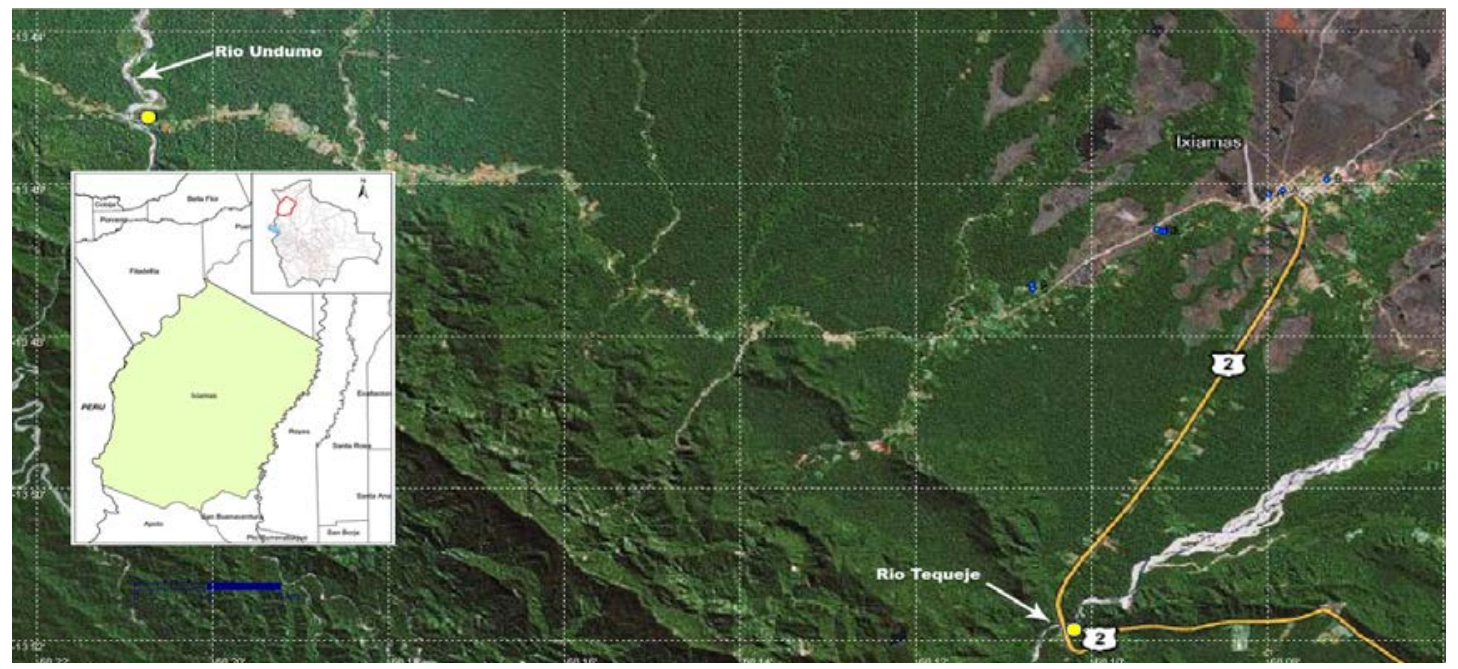

Figura 1. Imagen satelital de la localidad de Ixiamas y sus alrededores, donde se observan los puntos de muestreo, incluidos los puntos del Río Tequeje y del Río Undumo

\section{Preparación de muestras}

Los frutos de Asaí se prepararon tomando la pulpa y la cáscara, mezclándolos hasta formar una masa homogénea con las que se realizó cada una de las determinaciones.

\section{Determinaciones físicas, fisicoquímicas y químicas en frutos y Palmito (Tabla 2)}

Downloadable from: Revista Boliviana de Química. http://www.bolivianchemistryjournal.org
226

Volumen 38 Nº5. Año 2021

http://www.scribd.com/bolivianjournalofchemistry 
1. Características del fruto: Se observó forma, color y tamaño del fruto. Se determinó la masa, separando y pesando cada una de sus partes como: fruto, pulpa, cáscara y semilla. Posteriormente se calculó el porcentaje de estos componentes.

2. Humedad: Para determinar la humedad se utilizó el método de secado que propone Kirk, et al. [10], a una temperatura de $70^{\circ} \mathrm{C}$, empleando una estufa de secado Modelo ED-53, Binder.

3. pH: Se midió en la pulpa comestible [10], utilizando un pH-meter CG-840 Schott con electrodo Inab 427 Mettler Toledo.

4. Lípidos: Se determinó utilizando el método propuesto por Pearson [11].

5. Nitrógeno total y estimación de Proteína bruta: Se utilizó el Método Kjeldahl semimicro propuesto por Pearson [11] utilizando para la digestión mezcla reactiva de selenio. Se libera el amoniaco por el agregado de hidróxido de sodio. El destilado se recibió en una mezcla de ácido bórico-indicador (rojo de metilo y verde de bromocresol), posteriormente se tituló con ácido clorhídrico valorado [12]. Se utilizó el factor de 6.25 para convertir el nitrógeno en proteína bruta [8].

6. Cenizas y minerales: se determinaron según método por Kirk, et al. [10], que consiste en preparar una muestra y colocarla en un crisol de sílice, previamente calcinado y enfriado antes de pesarlo. El crisol y su contenido se incineran primero en forma suave hasta que se carboniza la muestra y luego a una temperatura entre $550^{\circ} \mathrm{C} \mathrm{a}$ $600^{\circ} \mathrm{C}$, en un Horno Mufla 1500 Furnace Termolyne. Se saca de la Mufla, se enfría y se pesa, la diferencia en porcentaje es la ceniza. Posteriormente a la ceniza, se le hace un tratamiento con ácido clorhídrico, luego se lleva a sequedad, para posteriormente precipitar la sílice. Se filtra, el residuo se vuelve a calcinar y se pesa, reportando el contenido de sílice en porcentaje [12]. El filtrado se afora a determinado volumen y se determinan los contenidos totales de los diferentes elementos [13].

7. Calcio, magnesio, sodio, potasio, hierro, cinc, cobre y manganeso: Se determinaron a partir del último filtrado aforado en la determinación de cenizas. Se utilizó la técnica de Absorción Atómica para cada elemento, realizando el tratamiento respectivo, según el Manual de Absorción Atómica de los equipos utilizados [14]. Los equipos utilizados fueron los Modelos AAnalyst 100 y AAnalyst 200 de la Perkin Elmer Instrument.

8. Fósforo: Se analizó a partir del último filtrado de la determinación de cenizas [6], empleando un Método Colorimétrico [10], midiendo en un Espectrofotómetro Hitachi modelo 200-20.

9. Carbohidratos: Se calculó por diferencia: $100 \%$ menos los porcentajes de lípidos, proteínas y cenizas [12], todos los contenidos calculados en muestra secada a $70^{\circ} \mathrm{C}$.

10. Valor Energético: Se calculó utilizando factores de conversión [13], [15], de la siguiente manera: Valor Energético $(\mathrm{kcal})=4 \mathrm{x}(\%$ de proteínas $)+4 \mathrm{x}(\%$ de carbohidratos $)+9 \mathrm{x}(\%$ de lípidos). Está expresado en $\mathrm{kcal} / 100 \mathrm{~g}$ de muestra seca (MS), en este estudio las muestras fueron desecadas a $70^{\circ} \mathrm{C}$

11. Para la determinación de la capacidad antioxidante se utilizó método FRAP que evalúa la capacidad antioxidante de una muestra de acuerdo con su capacidad para reducir el complejo amarillo de Fe3+-TPTZ (2, 4, 6-tripiridil-s-triazina) al complejo azul de Fe2+-TPTZ. Este último complejo tiene un máximo de absorbancia a una longitud de onda entre 590-595 nm, como está descrito por Peñarrieta et al. 2008 [16].

\section{Presentación y degustación del refresco del fruto de la palmera Asaí}

Se elaboraron productos alimenticios en base a la parte comestible del fruto de palmeras nativas, en este caso la palmera Asaí como es la producción de refrescos. (dirigidos a grupos etéreos de alto riesgo nutricional y realizar la evaluación sensorial correspondiente, a fin de proponer un aprovechamiento industrial de la parte comestible de las especies vegetales de la Amazonía paceña).

De esta manera se contribuye a mejorar los índices de nutrición de los lugareños, pues podrían trabajar en la producción de estos frutos exóticos, en actividades de comercio tanto de los frutos como de los productos elaborados. De esta manera se contribuiría a la generación de una agricultura sostenible, promoviendo la conservación de estos recursos [18,19].

\section{Producto alimenticio elaborado: refresco de Asaí}

El refresco de Asaí fue preparado de la siguiente manera.
$1000 \mathrm{~g}$
$1000 \mathrm{~mL}$
fruto de Asaí
$1000 \mathrm{~mL}$
agua de remojo
líquido obtenido 
Galia chávez cury et al. RBQ Vol.38, No.5, pp. 223-232, 2021

Se remojó las semillas de Asaí en agua tibia $\left(45^{\circ} \mathrm{C}\right)$, por el lapso de 12 horas, hasta que la corteza quedó suave y el agua adquirió un color rojo vino. El producto fue filtrado en un cedazo de malla

Análisis sensorial estándar, características organolépticas, prueba de aceptabilidad hedónica

Características organolépticas, variables evaluables

- Líquido color vino ligeramente opaco

- Olor leñoso

- Sabor leñoso, tipo ciruela.

- Aceptación agradable. Me gusta, a me gusta mucho

\section{Metodología}

El análisis sensorial estándar consiste en cuantificar la magnitud o grado de aceptación/rechazo del producto. Esta metodología es ampliamente aceptada a nivel internacional en estudios de investigación sensorial pre-lanzamiento de nuevos productos alimenticios al mercado o bien cuando se tiene planeado el cambio de algún ingrediente en productos en actual comercialización y habitual consumo [20].

Para la realización de esta prueba, se emplea un conjunto de degustadores voluntarios conocedores de la metodología con un sentido del gusto y olfato de probada sensibilidad, a quienes se les asigna aleatoriamente las muestras en estudio para que expresen su opinión con base en un formato estándar de encuesta (boleta/formulario).

Con el propósito de mantener la sensibilidad organoléptica con la menor alteración posible ante pruebas sucesivas, los degustadores disponen de agua para enjuague bucal sistemático intercalando la degustación de muestras, de manera de no confundir ni enmascarar sabores/olores propios de cada producto.

La prueba aleatoria de las muestras para la aplicación de un diseño experimental por bloques permite realizar un análisis objetivo de las muestras en estudio en el cual los efectos de preferencias individuales no sesguen el análisis.

Se hace la entrega de muestras en estudio en bandejas y vasos idénticos de color transparente, cuya identificación numerada aleatoria corresponde a un diseño ciego simple (el degustador no sabe a priori que numeración corresponde a qué tipo de muestra, patrón o estudio).

El orden de degustación, el enjuague bucal pre e inter evaluación, la temperatura, iluminación, olores y ruidos ambientales se mantuvieron bajo control y responsabilidad directa de la encargada del análisis sensorial, de manera tal de minimizar los sesgos que pudiesen producirse a causa de estas variables externas.

Una vez realizada la degustación de las muestras, el degustador tiene que llenar la boleta/formulario de evaluación inmediatamente después, antes de someterse a la siguiente prueba con otra muestra.

\section{Procedimiento}

Las muestras fueron evaluadas por 30 degustadores voluntarios a quienes se les entregó una bandeja con una o dos muestras de acuerdo a la prueba en ejecución y el producto a evaluar, un vaso de agua para el enjuague de la boca y un vaso para escupir la muestra y el agua. En todas las muestras se les pidió evaluar el grado de aceptación o rechazo, se anotaron los comentarios sobre las características organolépticas de aspecto general, color, olor, sabor y textura.

\section{RESULTADOS Y DISCUSIÓN}

\section{Características físicas de la planta de Asaí (frutos y palmito)}

Plantas aproximadamente entre 15 a $20 \mathrm{~m}$ de alto. Fruto de color negro cuando está maduro, tiene forma esférica entre 1,1 a 1,3 cm de diámetro. Pulpa jugosa, de color morado; endocarpio duro. Las semillas esféricas aproximadamente de $1 \mathrm{~cm}$ de diámetro. La masa del fruto variaba de 0,9 a 1,9 g, el porcentaje de pulpa y cáscara variaba de 21,4 a $40,0 \%$.

El palmito de color blanco que llegó al laboratorio tenia de 24 a $30 \mathrm{~cm}$ de largo, de 4 a $5 \mathrm{~cm}$ de diámetro y una masa entre 400 a $500 \mathrm{~g}$.

\section{Resultados de análisis en muestras desecadas a $70^{\circ} \mathrm{C}$ (Tablas 2 y 3 )}

Downloadable from: Revista Boliviana de Química.

http://www.bolivianchemistryjournal.org
228

http://www.scribd.com/bolivianjournalofchemistry 
Tabla 2. Resultados de análisis bromatológicos, macro y micronutrientes en muestras desecadas a $70^{\circ} \mathrm{C}$, indicando los valores de $n$ y $\mathrm{cV}$

\begin{tabular}{rcccccc}
\hline PARÁMETROS & Asa 1 & Asa 2 & Asa 3 & Pal 1 & Pal 2 & Pal 3 \\
\hline *Valor energético (kcal) & 515.4 & 515.4 & 515.4 & 361.8 & 356.0 & 349.7 \\
***Agua (\%) & 29.4 & 29.4 & 29.4 & 90.2 & 89.2 & 89.3 \\
CV (\%) & 1 & 1 & 1 & $<1$ & $<1$ & $<1$ \\
Nitrógeno (\%) & 1.35 & 1.40 & 1.37 & 3.42 & 5.61 & 3.60 \\
CV (\%) & 3 & 1 & 3 & 1 & 2 & 3 \\
Proté́nas (\%) & 8.4 & 8.8 & 8.6 & 21.4 & 35.1 & 22.5 \\
Lípidos (\%) & 24.4 & 24.4 & 24.4 & 2.56 & 1.64 & 1.08 \\
CV (\%) & 5 & 5 & 5 & 5 & $<1$ & 2 \\
**Carbohidratos (\%) & 65.5 & 65.2 & 65.4 & 63.3 & 50.2 & 62.5 \\
Ceniza (\%) & 1.65 & 1.63 & 1.64 & 127 & 13.1 & $\mid 3.9$ \\
CV (\%) & 4 & 2 & 1 & $<1$ & 2 & 2 \\
Sílice (\%) & 0.65 & 0.42 & 0.49 & 1.14 & 1.53 & 1.61 \\
CV (\%) & 3 & 3 & $<1$ & $<1$ & 2 & 1 \\
Ca (mg/100 g MS & 114.0 & 117.7 & 116.9 & 459.8 & 1121.3 & 1176.3 \\
CV (\%) & 5 & $<1$ & $<1$ & 4 & 5 & 3 \\
Mg (mg/100 g MS & 54.6 & 98.7 & 92.3 & 611.4 & 1093.3 & 1047.0 \\
CV (\%) & 6 & 2 & 1 & 4 & 2 & 5 \\
Na (mg/100 g MS & 21.4 & 4.3 & 5.5 & 9.5 & 8.9 & 33.2 \\
CV (\%) & 2 & 5 & 5 & 4 & 6 & 2 \\
K (mg/100 g MS & 462.4 & 646.6 & 444.0 & 2211.5 & 3988.6 & 4142.5 \\
CV (\%) & 7 & $<1$ & 2 & 1 & 1 & \\
P (mg/100 g MS & 57.4 & 58.2 & 59.4 & 301.2 & 364.9 & 298.6 \\
CV (\%) & 6 & 2 & 2 & 3 & 4 & 4 \\
Fe (mg/100 g MS & 39.5 & 30.5 & 31.6 & 44.0 & 51.5 & 98.0 \\
CV (\%) & 4 & 2 & $<1$ & 5 & 3 & 5 \\
Zn (mg/100 g MS & 12.1 & 14.5 & 14.5 & 145.7 & 245.5 & 217.7 \\
CV (\%) & 5 & 2 & 5 & 3 & 1 & 3 \\
Cu (mg/100 g MS & 14.2 & 13.2 & 13.4 & 10.9 & 18.1 & 25.4 \\
CV (\%) & 2 & $<1$ & 2 & 1 & $<1$ & 5 \\
Mn (mg/100 g MS & 208.4 & 236.1 & 238.1 & 1516.2 & 2605.0 & 3620.0 \\
CV (\%) & 8 & $<1$ & 5 & $<1$ & 1 & 3 \\
\hline Cálculos empleando factores, ** Cálculos por diferencia, *** El porcentaje agua es con respecto a la masa de muestra fresca \\
& & & & & & \\
\hline
\end{tabular}

Tabla 3. Otros resultados de análisis realizados en fruto y palmito de Asaí

\begin{tabular}{|c|c|c|}
\hline pH & En Fruto: $5.1 \quad C V<1$ & En Palmito: 6.0 CV $<1$ \\
\hline${ }^{*}$ Vitamina A & $\begin{array}{l}\text { En Fruto: } 442.47 \mathrm{ug} / 100 \mathrm{~g} . \\
\text { muestra }\end{array}$ & $\begin{array}{l}\text { En Palmito: } 0.0 \text { ug/100 g } \\
\text { muestra }\end{array}$ \\
\hline${ }^{*}$ Vitamina C & $\begin{array}{l}\text { En fruto: } 0.0 \mathrm{ug} / 100 \mathrm{~g} \text {. } \\
\text { muestra }\end{array}$ & $\begin{array}{l}\text { En palmito: } 0.0 \mathrm{ug} / 100 \mathrm{~g} \\
\text { muestra }\end{array}$ \\
\hline \multirow{2}{*}{$\begin{array}{l}\text { Caracterización del } \\
\text { aceite del fruto de Asaí } \\
\text { (pulpa y cáscara) }\end{array}$} & $\begin{array}{l}\text { Densidad }=0.9340 \mathrm{~g} / \mathrm{mL} \\
\mathrm{CV}<1\end{array}$ & Índice de acidez (IA) = 21.8 \\
\hline & $\begin{array}{l}\text { Acidez (\% masa de ácido } \\
\text { Oleico): } 11.00 \%, \mathrm{CV}<1\end{array}$ & \\
\hline
\end{tabular}




\section{Evaluación del contenido de macro y micronutrientes en frutos y palmito de asaí (Tabla 2)}

Agua: Los porcentajes de agua en el fruto, varían de 13.2 a 58.3 \%. Los datos en palmito fluctúan de 89.2 a $90.2 \%$.

Proteínas: En el fruto oscilan de 8.4 a 8.8 \% y el palmito muestra datos muy altos de 21.4 a $35.1 \%$.

Lípidos: Los porcentajes de lípidos en frutos oscilan de 24.4 a $53.4 \%$, indicando que son especies con alto potencial en la extracción de aceite. En el palmito oscilan entre 1.08 a $2.56 \%$.

Carbohidratos: En frutos varían de 65.2 a $65.5 \%$. En el palmito varia de 50.2 a 63.3 \%.

Valor Energético: Los frutos muestran un valor de 515.4 kcal/100 g MS y en palmito los valores fluctúan de 349,7 a $361.8 \mathrm{kcal} / 100 \mathrm{~g}$ MS.

Cenizas: Los resultados en el fruto varían de 1,63 a 1.65 \% en muestras de Palmito los valores oscilan de 12,7 a $13,9 \%$.

Calcio: Los valores en fruto oscilan de 114.0 a $117.7 \mathrm{mg} / 100 \mathrm{~g}$ MS y el palmito con valores altos de 459,8 a $1176,3 \mathrm{mg} / 100 \mathrm{~g}$ MS.

Potasio: Los contenidos en fruto varían de 444.0 a 646.6 mg/100 g MS, el palmito con valores altos de 2211,5 a $4142,5 \mathrm{mg} / 100 \mathrm{~g}$ MS.

Magnesio: Los datos en el fruto varían de 54.6 a 98.7 mg/100 g MS, excepto en el palmito con los resultados más altos de 611,4 a 1093,3 mg/100 g de MS.

Sodio: Los valores de sodio en el futo 4.3 a $21.4 \mathrm{mg} / 100 \mathrm{~g}$ de MS, en palmito varían de 8,9 a 33,2 mg/100 g de MS.

Fósforo: Los resultados en fruto oscilan de 57.4 a $59.4 \mathrm{mg} / 100 \mathrm{~g}$ de MS, siendo el palmito el que muestra los valores más altos de 298,6 a 364,9 mg/100 g de MS.

Hierro: Los contenidos en fruto fluctúan de 30.5 a 39.5 mg $/ \mathrm{kg}$ MS y en palmito varían 44,0 a 98,0 mg/kg MS.

Cinc: Los valores de cinc para el fruto varían de 12.1 a 14.5 mg/kg MS y en el palmito de 145.7 a 245.5 mg $/ \mathrm{kg}$ MS.

Cobre: Los contenidos de cobre para el fruto varían de 13.2 a $14.2 \mathrm{mg} / \mathrm{kg}$ MS y en palmito de 10.9 a $25.4 \mathrm{mg} / \mathrm{kg}$ MS.

Manganeso: Los contenidos varían de 208.4 a 238.1 mg/kg MS y en palmito oscila de 1516.2 a 3620.0 mg/kg MS

Como se puede observar son alimentos naturales muy ricos en nutrientes. El palmito muestra los valores más altos en: Proteínas, calcio, magnesio, potasio, fósforo, manganeso, cinc. El fruto muestra valores relativamente altos comparados con otros frutos, en proteínas, así como también en calcio, magnesio y potasio. Algo importante en los frutos se determinó Vitamina A [21-23].

La actividad antioxidante fue determina en las muestras de Asaí . El valor promedio determinado fue de 16,6 $\mu$ moles de Trolox equivalente por gramo de muestra fresca por el método FRAP con sus siglas en inglés Ferric Reduction Antioxidant Power Estos valores nos muestran s que el Asaí presentan valores altos en comparación con otras palmeras.

\section{Resultados de la prueba hedónica, análisis sensorial estándar}

Los resultados de la prueba hedónica que indican el grado de aceptabilidad o rechazo, representan a los valores obtenidos según la tabla utilizada que se presenta a continuación: 
Tabla 4. Resultados de la Prueba Hedónica que indican el grado de aceptabilidad o rechazo

\begin{tabular}{|c|c|}
\hline $\begin{array}{l}\text { GRADO DE ACEPTABILIDAD MEDIDO EN LA PRUEBA } \\
\text { HEDONICA }\end{array}$ & VALOR \\
\hline Me gusta MUCHO & 7 \\
\hline Me gusta & 6 \\
\hline Me gusta UN POCO & 5 \\
\hline Ni me gusta ni me disgusta (me es indiferente) & 4 \\
\hline Me disgusta UN POCO & 3 \\
\hline Me disgusta & 2 \\
\hline Me disgusta MUCHO & 1 \\
\hline
\end{tabular}

\section{Puntaje obtenido en la aceptabilidad por fruto y producto}

En el siguiente cuadro se presenta el puntaje obtenido del grado de aceptabilidad del producto evaluado con la prueba hedónica.

Tabla 5. Puntajes obtenidos en la aceptabilidad del refresco de Asaí

\begin{tabular}{ccc}
\hline Fruto & Producto & ACEPTABILIDAD \\
\hline Asaí & 1. Refresco & 6,62 \\
\hline
\end{tabular}

\section{CONCLUSIÓN}

Tanto el palmito con el Asaí son alimentos naturales muy ricos en nutrientes. El palmito presentó los valores más altos en: Proteínas, calcio, magnesio, potasio, fósforo, manganeso, cinc. El fruto muestra valores relativamente altos comparados con otros frutos, en proteínas, así como también en calcio, magnesio y potasio. Algo importante en los frutos se determinó Vitamina A.

Respecto de los valores de actividad antioxidante el Asaí presentó valores altos en comparación con otras bayas [24] probar.

La bebida elaborada tuvo buena aceptación por parte de los degustadores entrenados a quienes se les hizo

Por el número de respuestas obtenidas los resultados reflejan el grado de aceptación del consumidor potencial siendo éste adulto o niño.

\section{REFERENCIAS}

1. Montes de Oca, I. Geografía y Recursos Naturales de Bolivia, Edobol, 3ra edición, 1997, La Paz, Bolivia.

2. Boero, H. Bolivia Mágica, Tomo I, Editorial Vertiente, 1993, La Paz, Bolivia.

3. Hoskins, M. 1990, Las actividades forestales en la alimentación, Unasylva, 41(1), 3-13.

4. Moraes, R.M. 1996, Bases para el Manejo Sostenible de Palmeras Nativas de Bolivia, Ministerio de Desarrollo Sostenible y Medio Ambiente, Programa de las Naciones Unidas para el Desarrollo, Tratado de Cooperación Amazónica, Proyecto Aprovechamiento Sostenible de la Biodiversidad Amazónica, La Paz, Bolivia.

5. Ministerio de Salud y Deportes (MSD). 2010, Programa de Desnutrición Cero 2007-2011, Bolivia.

6. Arze, R.M., San Miguel, J. L. 2005, Punto de corte para definir anemia en mujeres embarazadas residentes de gran altitud, MSD, Fac. de Medicina, INSAD, Carrera Nutrición y Dietética, PMA, La Paz, Bolivia.

7. Villachica, H., de Carvalho, J. E.U., Mercado, R.S., Izquierdo, J., Egg, A. B. 1996, Frutales y hortalizas promisorios de la Amazonia, Tratado de Cooperación Amazónica, Secretaría Pro-Tempore, Lima, Perú, 44, 367.

8. Badui, S. Química de los alimentos, Longman de México Editores, S. A. de C. V., $3^{a}$ edición, 1999, México.

9. Vásquez, R., Coimbra, G. Frutas Silvestres Comestibles de Santa Cruz, Editorial FAN, $2^{\mathrm{a}}$ Edición, 2002, Santa Cruz, Bolivia.

10. Kirk, R., Sawyer, R., Egan, H. Composición y Análisis de Alimentos de Pearson, Compañía Editorial Continental S.A., 2a ed. en español, 1996, México.

11. Pearson, D. Técnicas de Laboratorio para Análisis de Alimentos, Editorial Acribia, $1^{\mathrm{a}}$ ed. en español, 1993, España.

12. Official Methods of Analysis, Food Composition; Additives; Natural Contaminants, 1990, 15th Edition, Volume Two. Association of Official Analytical Chemists, Inc., Arlington, Virginia, USA.

13. Adrian, J., Potus, J., Poiffait, A., Dauvillier, P. Análisis Nutricional de los Alimentos, Editorial Acribia, $1^{\text {a }}$ ed. en español, 2000, España.

14. Perkin Elmer. 2000, Analytical Methods for Atomic Absortion Spectrometry.

15. Jackson, M.L. Análisis Químico de suelos, Ediciones Omega, $3^{\mathrm{a}}$ ed., 1976, España.

Downloadable from: Revista Boliviana de Química. http://www.bolivianchemistryjournal.org
231

Volumen 38 Nº5. Año 2021

http://www.scribd.com/bolivianjournalofchemistry 
ISSN 2078-3949 Rev. Bol. Quim. Electronic edition

Galia Chávez Cury et al. RBQ Vol.38, No.5, pp. 223-232, 2021

16. Peñarrieta, J.M., Alvarado, J.A., Åkesson B., Bergenståhl, B. 2008, Total antioxidant capacity and content of flavonoids and other phenolic compounds in canihua (Chenopodium pallidicaule): An Andean pseudocereal, Mol. Nutr. Food Res, 52(6), $708-717$. DOI:10.1002/mnfr.200700189

17. Hernández, M., Sastre A. Tratado de Nutrición, Ediciones Díaz de Santos, S. A., $1^{\text {a }}$ ed. en español, 1999, España.

18. Larmond, E. 1977, Laboratory methods for sensory evaluation of food, Research Branch, Department of Agriculture, Ottawa, Publication 1637, Canada.

19. Pedrero, D.L., Pangborn, R.M. Evaluación sensorial de los alimentos - métodos analíticos; ed. alhambra mexicana, $1^{\mathrm{a}}$ ed. $2^{\mathrm{a}}$ reimpresión, 1990, Mexico.

20. Cruz-Dominguez, L., Utrilla-Estrada, B.A., Flores-Guillén, L.E., García-Parra, E., López-Zúñiga, E.J., Vela-Gutiérrez, G. 2016, Evaluación nutricional y sensorial de un alimento a base de atún y soya enriquecido con vitaminas y minerales, Rev. Chilena de Nutrición, 43(4), 388-393. DOI: http://dx.doi.org/10.4067/S0717-75182016000400008

21. Ministerio de Salud y Deportes. 2007, Recomendaciones de energía y de nutrientes para la población boliviana, $1^{\text {a }}$ ed., Publicación 29, La Paz, Bolivia.

22. IBNORCA. 1977, Aceites y grasas, determinación de la acidez, N. B., 166-177.

23. Araujo-Murakami, A. y Zenteno, F. 2006, Bosques de los Andes Orientales de Bolivia y sus especies útiles, Botánica Económica de los Andes Centrales (M. Moraes, R., B. Ollgaard, L., P Kvist, F. Borchsenius \& H. Balslev, Eds.), Universidad Mayor de San Andrés, La Paz, Bolivia, 146-161.

24. Peñarrieta, J.M., Alvarado, J.A., Bergenståhl, B., Åkesson, B. 2009, Total antioxidant capacity and content of phenolic compounds in wild Strawberries (Fragaria vesca) collected in Bolivia, Int. J. Fruit Sci., 9(4), 344-359. DOI: https://doi.org/10.1080/15538360903378526 